International Journal of Pure and Applied Mathematics

Volume 114 No. 1 2017, 113-132

ISSN: 1311-8080 (printed version); ISSN: 1314-3395 (on-line version)

url: http://www.ijpam.eu

doi: 10.12732/ijpam.v114i1.10

ijpam.eu

\title{
SYNCHRONIZATION AND CHANGES IN VOLATILITIES IN THE LATIN AMERICAN'S STOCK EXCHANGE MARKETS
}

\author{
G. Cabrera ${ }^{1}$, S. Coronado ${ }^{2}$, O. Rojas ${ }^{3}$, F. Venegas-Martínez ${ }^{4}$ § \\ 1,2Departamento de Métodos Cuantitativos \\ Centro Universitario de \\ Ciencias Económico Administrativas \\ Universidad de Guadalajara, MÉXICO \\ ${ }^{3}$ Escuela de Ciencias Económicas y Empresariales \\ Universidad Panamericana \\ Campus Guadalajara, MÉXICO \\ ${ }^{4}$ Escuela Superior de Economía \\ Instituto Politécnico Nacional \\ MÉXICO
}

\begin{abstract}
In this paper we study a possible synchronization in volatility changes for some Latin America's stock exchange indexes. We also add the S\&P 500 index to the analysis. We suggest a heterogeneity Markov switching model to capture changes in volatilities over time. To solve the problem of uncertainty in modeling each index, we suggest the Bayes Factor to identify the best Markov switching specification as the number of states, if any. We found that, all the daily growth rates for each index are well characterized by low, medium and high volatilities in different periods of time. We suggest some measures of synchronization based on the concordance by the changes in volatilities between the indexes. We show that, the Mexican, Chilean and the S\&P 500 indexes are closer to each other than the rest
\end{abstract}

AMS Subject Classification: C22, G15

Key Words: synchronization, Markov process, financial markets

\section{Introduction}

Investing in emerging markets is important for investors, as they present high returns, which therein carries greater risk; such as the return on investment, abnormal returns, high volatilities, structural changes, low correlation with developed markets. Studying these characteristics allows portfolio risk to be reduced, see [2], [21], [28] and [31].

Received: $\quad$ February 7, 2017

Revised: $\quad$ March 21, 2017

Published: $\quad$ April 20, 2017

(c) 2017 Academic Publications, Ltd.

url: www.acadpubl.eu 
At present there has been a financial integration between different economies, which are not exempt from economic and/or financial shocks internally or externally, which leads to periods of crisis and / or contagion, see [16] and Zouhair, 2013. In fact, during periods of calm, there are low yields, which allows the portfolio to diversify depending on the attitude of the investor towards risk, but in turbulent periods the correlation increases, so diversification in the portfolio is difficult [?]. Due to the above, the economic and/or financial series have behaviors characterized by high and low volatilities, during certain periods of time.

A classic nonlinear time series model that show abrupt changes over time is the one proposed by [17]. This model suggests that mean and variance are subject to change of state and explains the transition from one state to another. Different applications to economic and/or financial series have been made of this model to emerging countries and in particular to some countries in Latin America, see [3] and [7].

In this sense, studies on Latin American countries, some related to some United States index, we can mention the work of [11], who suggest an Autoregressive Conditional Heteroskedasticy Switch (SWARCH) with two states for The equity indexes, at the same time determine the synchronization through different combinations of state changes between pairs of countries. In this same line is the work of [3], who study periods of high volatility in stock markets through a SWARCH model. [7], studies the stock market correlation between countries by means of two models: Dynamic Conditional CorrelationGeneralized Autoregressive Conditional Heteroskedasticity (DCC-GARCH) and a SWARCH model, without going into synchronization.

Another example is the study by [10], who apply a non-parametric model to determine the phase of cycles for the six-country stock market, which allows them to determine where they change from expansion to recession and viceversa determining the points of change of passing of a regime, supported by the calculation of the concordance index Harding, 2000; Harding, 2002; Harding, 2003. On the other hand, Aiolfi, 2011, study business cycles, using different economic and financial variables, using a non-parametric common factor model and using the same concordance index. Dufrenot, 2011 study the effect of the crisis suppresses in some Latin American countries in relation to other financial indicators of the United States, applying a Time-Varing Probability Markov Switching (TVPMS) model. There is also the study by [28], who uses a heterogeneous regime switching model (HRSM), to determine the synchronization and co-movement between stock market prices.

In this work, we apply the methodology of [15], based on Bayesian meth- 
ods to simulate the marginal likelihood of mixture univariate models, which allows discrimination between models through Bayes Factor. Unlike the literature cited above, this methodology has not been applied to the study of the stock market of Latin American countries. In general, the specification of the model as the number of states has been exogenous, however we endogenously find three states characterized by changes in volatility. We also calculated the agreement between Harding \& Pagan to determine the correlation between the Latin American countries and the SP500, as well as determining the days of high, medium and low volatility across of the conditional probabilities of the state indicators.

The structure of the paper is as follows: in Section 2 we present the data to be used in the empirical study; in Section 3 we show the methods used to model the changes in volatility; in Section 4 we present the results of the empirical model, whereas in Section 5 we give some conclusions and possible lines of future research.

\section{Data}

We consider daily prices $p_{t}$ for the indexes of the stock exchange markets of the following five Latin American countries: Argentina's MERVAL (ARG), Bolivia's BBV (BOL), Brazil's BOVESPA (BRZ), Mexico's IPC (MEX), Chile's IPSA (CHI), and the S\&P 500 index for United States of America (SP500) for the period from June 28th, 1991 to June 28th, 2016 for a total of 6, 299 observations for each of the six indexes. Such dates are representative enough of different economic periods, including global and national financial crises, and therefore we expect to observe changes in volatility accordingly, see Fig. 1. As can be observed, there is significant co-movement between the markets and therefore we expect to find common dynamics in different time periods.

Prices are transformed into porcentual growth rates $y_{t}=100\left(\ln \left(p_{t}\right)-\right.$ $\left.\ln \left(p_{t-1}\right)\right)$. In Table 1 , summary statistics are presented for such growth rates $y_{t}$. Statistics are consistent with many stylized facts for time series, see [6], [29]. All series are leptokurtic, above the kurtosis for the normal distribution that is 3 , which is an indication of fat tails. The series also depart from normality according to the Jarque-Bera test, that rejects the null hypothesis of normality of the series at 1-percent level. Also note how ARG and BRZ have the highest standard deviation (2.31 and 2.57, respectively), while the rest of the markets have a standard deviation between 1.12 and 1.50. However, the range of all markets is between 46.05 for BRZ to 19.47 for CHI, an indication of periods of 


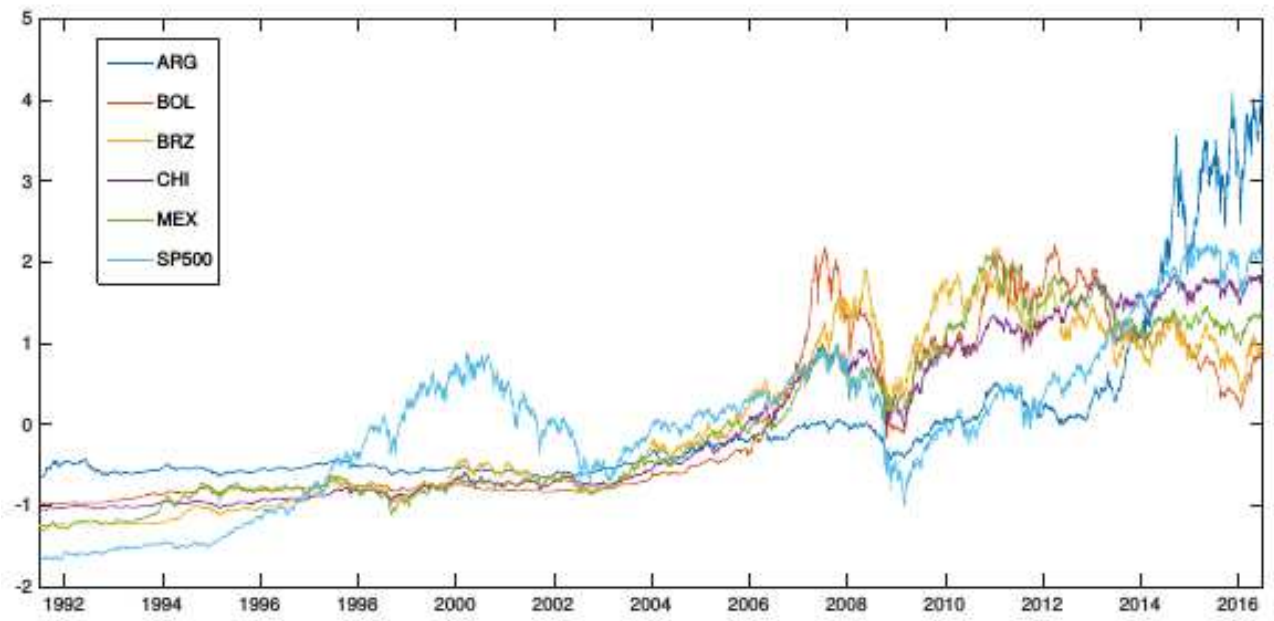

Figure 1: Time series plot of standardized prices $p_{t}$

different levels of volatility, as we will show in this paper.

Table 1: Descriptive statistics of $y_{t}$

\begin{tabular}{lrrrrrr}
\hline Statistics & ARG & BOL & BRZ & MEX & CHI & SP \\
\hline Observations & 6298 & 6298 & 6298 & 6298 & 6298 & 6 \\
Mean & 0.06 & 0.09 & 0.20 & 0.06 & 0.04 & \\
Min & -14.76 & -13.29 & -17.23 & -14.31 & -7.67 & - \\
Max & 16.12 & 12.82 & 28.82 & 12.15 & 11.80 & 1 \\
Range & 30.88 & 26.11 & 46.05 & 26.46 & 19.47 & 2 \\
Standard deviation & 2.31 & 1.50 & 2.53 & 1.50 & 1.12 & \\
Skewness & -0.13 & -0.02 & 0.49 & 0.00 & 0.19 & - \\
Kurtosis & 7.23 & 10.94 & 10.68 & 9.63 & 10.10 & 1 \\
Jarque-Bera & 4703.37 & 16537.72 & 15747.180 & 11535.88 & 13261.92 & 2040 \\
ADF & $-73.08^{* * *}$ & $-41.26^{* * *}$ & $-76.58^{* * *}$ & $-71.570^{* * *}$ & $-51.44^{* * *}$ & -60.57 \\
RALS & $-83.53^{* * *}$ & $-43.68^{* * *}$ & $-80.36^{* * *}$ & $-76.50^{* * *}$ & $-53.91^{* * *}$ & -64.22 \\
\hline
\end{tabular}

Given that the models fitted to the data require the series to be integrated of order 0, $I(0)$, or stationary, we perform the Augmented Dickey, 1981 (ADF) test and the Residual Augmented Least Squares (RALS) test of [22], which is more robust since it does not require the series to be normally distributed. Both tests results give evidence of the stationarity of the series, rejecting the null hypothesis of non-stationarity (presence of a unit root) at the 1-percent 
level, where the lag length was carried using the Bayesian information criteria (BIC).

\section{Methods}

Following [14], we model the changes in volatility of the daily growth rates $y_{t}$ using a Markov Switching Autoregressive model (MSAR), where the full set of parameters is state-dependent, given by

$$
\phi_{s_{t}}(L) y_{t}=\zeta_{s_{t}}+\sigma_{s_{t}} \varepsilon_{t}
$$

where $\varepsilon_{t} \sim \mathcal{N}(0,1) ; \phi_{s_{t}}(L)$ is a polynomial of order $p$ and $L$ is the lag operator, subject to different states $s_{t} ; \zeta_{s_{t}}$ is the state-dependent intercept, and $\sigma_{s_{t}}$ captures the state-dependent volatility due to the error term. The statedependency of the parameters is captured by the hidden indicator $s_{t}$, which follows a discrete-time Markov process of order one, i.e., the transition probability from state $k$ to state $l$ is given by: $\xi_{k l}=\operatorname{Pr}\left(s_{t}=l \mid s_{t-1}=k\right)$ for all $t=1, \ldots, T$ and the states $k, l \in\{1, \ldots, K\}$. We denote the set of parameters and the hidden indicator of the states by $\Psi=(\psi, S)$ where $S=\left(s_{1}, \ldots, s_{T}\right)$ and $\psi=\left(\phi_{k}, \zeta_{k}, \sigma_{k}, \xi_{k l}\right)$. According to all possible combinations of models and parameters, there are $K(p+1)+p(K-1)$ possible models. Thus, we would like to find the model of best-fit to data.

In particular, if $K=1$ the MS specification leads to a $V A R(p)$. If $K=2,3$ we denote $M S I A R(p)$ when only the intercept and variance of the error term are state dependent and, $M S A R(p)$ when all the parameters are state dependent.

Using the bridge sampling technique ([27] and [14] and [15]), we simulate the Marginal Likelihood (ML) of each possible model by running unrestricted MCMC sampling ${ }^{1}$. In this way we can compare model $i$ from model $j$ using the Bayes Factor given by

$$
B F_{i, j}=\frac{L\left(y \mid M_{j}\right)}{L\left(y \mid M_{i}\right)}=\frac{p\left(M_{j} \mid y\right)}{p\left(M_{i} \mid y\right)}
$$

where $L\left(y \mid M_{j}\right)$ is the ML of data $y$ conditioned to model $j$ and $p\left(M_{j} \mid y\right)$ is the probability of model $j$ conditioned to data $y$. Note that there is no prior probability assigned to any model $j$, i.e., $p\left(M_{j}\right)=p\left(M_{i}\right)$. The marginal likelihood to the model $j, p\left(M_{j}\right)$, is given by the Bayes theorem as follows:

$$
p(y)=\frac{p\left(y \mid \theta_{j}\right) p\left(\theta_{j}\right)}{p\left(\theta_{j} \mid y\right)}
$$

\footnotetext{
${ }^{1}$ Unrestricted MCMC sampling means that there is no prior restriction to identify the state dependent parameters
} 
where $p\left(y \mid \theta_{j}\right)$ is the standard likelihood in classical econometrics, $p\left(\theta_{j}\right)$ is the prior of the parameters to the model $j$ and, $p\left(\theta_{j} \mid y\right)$ is the posterior density of the parameters conditional to the observed data. Hence, the model with the highest ML is preferred. Since we are interested in knowing the probabilities for each model to the best, we will infer the value of the probability from this ML ratio.

Even though the prior and likelihood densities ares easy to be evaluated, the posterior does not since the Markov switching models leads mixture distributions associate with label switching problems. Therefore, simulation methods are used, see [15] for further details. Under the Bayes factor ratio, the model $j$ that leads the highest ratio over the rest would be preferred.

Once the best-fit model is chosen, we carry out a new MCMC for the best specification of the model by using some restrictions to identify all statedependent parameters. The smoothed probabilities are computed from the best-fit model and found according to the forward filtering backward smoothing algorithm, see [?].

The MCMC sampling based on a data augmentation scheme is typically used (Kim, 1998; Kaufmann, 2000; Frühwirth-Schnatter, 2001). In general, MCMC sampling to obtain (1), for switching Gaussian state space models suggests implementing a block sampler according to the following steps: 1) Sample $S$ conditional on $(\psi)$ and data from $p(S \mid \psi, y)$. 2) Sample $\psi$ conditional on $(S)$ from the complete data posterior density $p(\psi \mid S, y)$. 3) Permutation sampler. We repeat such process 6,000 times, discarding the first 1,000 to eliminate the dependency on initial conditions. However, convergence is reached with less than 5,000

The generation of the hidden indicator Markov chain is based on multimove Gibbs sampler suggested by Carter and [4] and [30], that samples jointly the whole path $S$. Sampling unknown parameters is straightforward since $(S)$ is treated as data and standard well known Bayesian methods can therefore be applied. Based on [14] (2004 and 2006). Note that, most of the densities are multimodal because state dependent parameters are present (mixture distributions). Then to explore the full mixture posterior distribution, it is necessary to force the MCMC scheme to visit appropriately the space of the parameters. That is, a balanced label switching is carried out by randomly selected permutation of the labeling.

Conclude each draw by selecting randomly one of $K$ ! permutations ${ }^{2}$ of the current labeling. This permutation is applied at all state dependent parameters

\footnotetext{
${ }^{2}$ if $K=2$ then permutations are $\{12,21\}$, if $K=3$ there are six permutations: $\{123,132,213,231,312,321\}$
} 
and the hidden indicator.

Now we turn to explain the Marginal likelihood by the bridge sampling estimator for model comparison. It is well known that posterior odds ratio leads to Eq. 2. Since there is no prior preference for some model, $p\left(M_{j}\right)=p\left(M_{i}\right)$. Hence, the model with the highest model likelihood $L\left(y \mid M_{j}\right)$ is preferred.

This likelihood is obtained by integrating the complete data likelihood $f(y \mid$ $\Psi)$ with respect to the prior of $p(\Psi)$ where $\Psi=(\psi, S)$ :

$$
L\left(y \mid M_{j}\right)=\int f(y \mid \Psi) p(\Psi) d(\Psi)
$$

Since analytical integration of (3) is not feasible, some approximation method should be used. [15] suggests a way to reduce the dimensionality integration problem. It is possible to integrate out the switching process $S$ and marginal likelihood is given by: ${ }^{3}$

$$
L(y)=\int L(y \mid \psi) p(\psi) d(\psi)
$$

Where a closed form of a likelihood $L(y \mid \psi)$ and prior $p(\psi)$ are available. Following [14] we estimate the marginal likelihood (4) since, it does not depends on Gaussianity nor linearity. See [14] to derive the optimal bridge sampling estimator as follows:

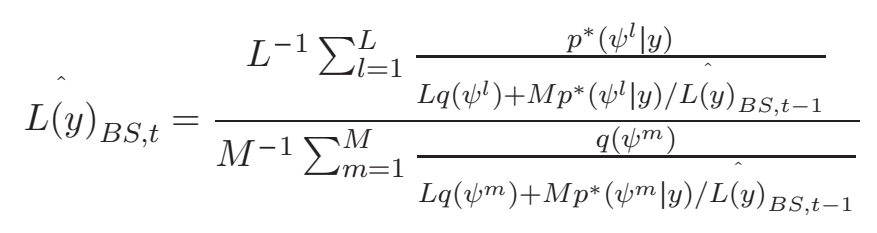

Iteration of (5) is initialized with $L(y)_{B S, 0}$ equals to either Importance Sampling (IS) or Reciprocal Importance sampling estimator (RI). See [15] for further details. follows:

The algorithm to compute $L(y)_{B S, t}$ is summarized in ch.5 p-152 ([15]) as

1. Simulation step: Run a MCMC sampler to obtain $\left\{\psi^{m}\right\}_{m=1}^{M}$ draws from posterior $p(\psi \mid y)$. Choose an importance density $q(\psi)$ and get i.i.d. $\left\{\psi^{l}\right\}_{l=1}^{L}$ draws.

\footnotetext{
${ }^{3}$ That is, by integrating out $S$ we have:

$L(y, \psi)=\int L(y, S, \psi) d S$

Finally: $L(y)=\int L(y \mid \psi) p(\psi) d(\psi)$
} 
2. Functional evaluation: Evaluate both the non-normalized posterior $p^{*}(\psi \mid$ $y$ ) and the importance density $q(\psi)$ at all draws from the posterior at all draws from the importance density.

3. Iteration: Use the functional values to determine the initial $L(y)_{B S, 0}$ and run (5) until convergence. It is important to notice that functional values are fixed $\left(L, M, p, q, q^{*}\right)$ to iterate (5) then convergence is very fast.

Now we turn to the choice of the importance density for switching state pace models. It is traditional to choose a normal importance density. However, the posterior density in Markov switching models is usually multimodal and a normal importance density is a bad choice. [14] suggest a random subsequence $n=1, \ldots, M_{L}$ of MCMC draws $\Psi^{m}, m=1, \ldots, M$ where $\Psi^{m}=(\psi, S)^{m}$ could be used to construct an importance density:

$$
q(\psi)=M_{L}^{-1} \sum_{n=1}^{M_{L}} p\left(\psi \mid y,(S)^{n}\right)
$$

and

$$
q(\psi)=M_{L}^{-1} \sum_{n=1}^{M_{L}} K_{\theta}\left(\theta \mid(\theta, S)^{n}, y\right)
$$

Where $K_{\theta}(\theta \mid \theta, y, S)$ is called the density of the transition kernel appearing in the unconstrained sampling. The weights of the mixture importance density are given by $p(\xi \mid y, S)$, and $p(\psi \mid y, S)$. Normalizing constants are available for all conditional densities.

Typically random subsequences $M_{L}<<M$ because equation (6) needs to be only a rough approximation of the posterior. This is based on averaging over the conditional densities, where the argument $(\psi)$ is fixed and the conditioning indicator process $S$ is sampled from unconstrained posterior switching between different ways of labeling as permutation sampler does.

Next, in order to measure synchronization between the different regimes of volatilities of the markets, we follow the Harding and Pagan approach to measure the perfect synchronization in markets $i$ and $j$, using a concordance Index $(C I)$ defined by

$$
C I_{i j}=\frac{1}{T} \sum_{t=1}^{T} I\left(y_{i t}=y_{j t}\right),
$$

where $I(\cdot)$ is an indicator function. This measure of synchronization suggested by Harding and Pagan is not a cross dynamic measures, in the sense that only 
the concordance in the same period of time is relevant to the magnitude of the measure.

\section{Results}

In Table 2 we present the results of the simulation of the ML using the bridge sampling technique as discussed in the previous section, i.e., $\log \left(L\left(y \mid M_{j}\right)\right)$ for $K=1,2,3$ states and $p=0,1,2$ autoregression lags. The ML suggests the best model that represents the data for each index. All of them are characterized by $K=3$ states and: $\operatorname{MSIAR}(2)$ for $\mathrm{ARG}, \operatorname{MSAR}(1)$ for $\mathrm{BOL}, \operatorname{MSAR}(0)$ for BRZ and MSIAR(2) for MEX, CHI and SP500.

Table 2: Log of the Marginal likelihood

\begin{tabular}{cccccccc}
\hline K & Model & ARG & BOL & BRZ & MEX & CHI \\
\hline 1 & AR(0) & $-14,656.57$ & $-11,780.75$ & $-15,203.42$ & $-11,779.31$ & $-9,874.14$ & - \\
1 & AR(1) & $-14,641.09$ & $-11,577.40$ & $-15,201.80$ & $-11,747.25$ & $-9,753.55$ & - \\
1 & AR(2) & $-14,638.06$ & $-11,572.79$ & $-15,205.59$ & $-11,746.21$ & $-9,757.06$ & - \\
2 & MSAR(0) & $-13,702.61$ & $-10,300.22$ & $-13,893.13$ & $-10,841.59$ & $-8,971.40$ & - \\
2 & MSAR(1) & $-13,695.74$ & $-10,151.34$ & $-13,896.94$ & $-10,814.47$ & $-8,859.69$ & - \\
2 & MSAR(2) & $-13,694.88$ & $-10,153.58$ & $-13,902.53$ & $-10,816.11$ & $-8,862.57$ & - \\
3 & MSAR(0) & $-13,613.72$ & $-10,153.70$ & $\mathbf{- 1 3 , 7 3 4 . 7 0}$ & $-10,729.83$ & $-8,840.72$ & - \\
3 & MSAR(1) & $-13,610.93$ & $-10,097.85$ & $-13,741.35$ & $-10,703.35$ & $-8,714.97$ & $-\varepsilon$ \\
3 & MSAR(2) & $-13,659.15$ & $-10,102.75$ & $-13,749.49$ & $-10,707.53$ & $-8,802.43$ & $-\varepsilon$ \\
2 & MSIAR(1) & $-13,690.65$ & $-10,149.76$ & $-13,896.69$ & $-10,812.69$ & $-8,858.31$ & $-\varepsilon$ \\
2 & MSIAR(2) & $-13,687.05$ & $-10,150.16$ & $-13,898.05$ & $-10,810.45$ & $-8,857.73$ & $-\varepsilon$ \\
3 & MSIAR(1) & $-13,604.24$ & $\mathbf{- 1 0 , 0 1 1 . 0 4}$ & $-13,739.25$ & $-10,699.85$ & $-8,713.20$ & $-\varepsilon$ \\
3 & MSIAR(2) & $\mathbf{- 1 3 , 6 0 0 . 9 2}$ & $-10,012.29$ & $-13,740.34$ & $\mathbf{- 1 0 , 6 9 7 . 7 8}$ & $\mathbf{- 8 , 7 1 1 . 3 5}$ & $-\mathbf{8}$ \\
\hline
\end{tabular}

Once the best model is chosen, we carry out a new MCMC for the best specification of the model by using the restrictions to identify the state dependent variance $\sigma_{1}^{2}>\sigma_{2}^{2}>\sigma_{3}^{2}$.

In Table 3, we present the posterior mean for each parameter to the bestfit model. The last three rows show the posterior steady-state probabilities for the different levels of volatility ${ }^{4}$. Given the high frequency of the data, some state dependent intercepts (therefore the state dependent mean) go to zero. The highest volatilities are for CHI (3.48) and BOL (3.38). The highest

\footnotetext{
${ }^{4}$ We denote the posterior steady-state probabilities as $\pi_{k}$ for $k=1,2,3$ for $P^{\prime} \pi=\pi$
} 
persistence of the state of high volatility are BRZ (0.98) and the SP500 (0.97) indexes. ARG presents the less persistence and therefore is the index with more volatility $(0.94,0.88$ and 0.81 persistence for high, medium and low volatility respectively). ARG and BRZ have more probability to stay in high volatility (0.19 and 0.16 respectively). CHI, MEX and SP500 stay in more than the $90 \%$ of the time in low and medium volatility; they seem to be the less risky and volatile indexes.

Table 3: Posterior means of the parameters of the MSIAR

\begin{tabular}{crrrrrr}
\hline Parameters & ARG & BOL & BRZ & MEX & CHI & SP500 \\
\hline$\zeta_{1}$ & -0.14 & 0.13 & 0.22 & -0.14 & -0.28 & -0.17 \\
$\zeta_{2}$ & -0.01 & 0.10 & -0.06 & -0.02 & 0.01 & -0.03 \\
$\zeta_{3}$ & 0.04 & 0.04 & -0.03 & 0.02 & 0.01 & 0.05 \\
$\phi_{1}$ & 0.06 & 0.23 & & 0.12 & 0.21 & -0.04 \\
$\phi_{2}$ & -0.01 & & & -0.04 & -0.03 & -0.03 \\
$\sigma_{1}$ & 1.97 & 3.38 & 1.88 & 2.39 & 3.48 & 2.38 \\
$\sigma_{2}$ & 1.01 & 1.41 & 0.85 & 1.22 & 1.26 & 1.00 \\
$\sigma_{3}$ & 0.48 & 0.67 & 0.50 & 0.59 & 0.62 & 0.49 \\
$\xi_{11}$ & 0.94 & 0.88 & 0.98 & 0.89 & 0.89 & 0.97 \\
$\xi_{21}$ & 0.01 & 0.03 & 0.01 & 0.01 & 0.01 & 0.01 \\
$\xi_{31}$ & 0.02 & 0.00 & 0.00 & 0.00 & 0.00 & 0.00 \\
$\xi_{12}$ & 0.06 & 0.12 & 0.01 & 0.11 & 0.10 & 0.03 \\
$\xi_{22}$ & 0.88 & 0.90 & 0.98 & 0.96 & 0.96 & 0.97 \\
$\xi_{32}$ & 0.17 & 0.04 & 0.01 & 0.02 & 0.02 & 0.02 \\
$\xi_{13}$ & 0.01 & 0.00 & 0.00 & 0.00 & 0.01 & 0.00 \\
$\xi_{23}$ & 0.11 & 0.07 & 0.01 & 0.03 & 0.03 & 0.02 \\
$\xi_{33}$ & 0.81 & 0.96 & 0.99 & 0.98 & 0.98 & 0.98 \\
$\pi_{1}$ & 0.19 & 0.10 & 0.16 & 0.05 & 0.03 & 0.09 \\
$\pi_{2}$ & 0.50 & 0.36 & 0.39 & 0.38 & 0.33 & 0.42 \\
$\pi_{3}$ & 0.31 & 0.55 & 0.45 & 0.57 & 0.65 & 0.49 \\
\hline
\end{tabular}

In Fig. 2, 3 and 4 we plot the growth rates versus the smoothed states probabilities of high, medium and low volatility, respectively. Most of the times (except for ARG) there is a good adjustment of the state dependent changes in the volatilities. That is, when the probabilities go to one, the volatility belongs to one state characterized, by low, medium and high volatility. If these probabilities go down, there might be a change in the volatility. The exceptional case is ARG, since is all the the states are the less persistence, is the most volatile index. That is, there are several changes in volatilities over 
time. The index goes from medium to low and medium to high very often. However, we would like to find out how related or synchronized are the markets under study, according to their volatilities.
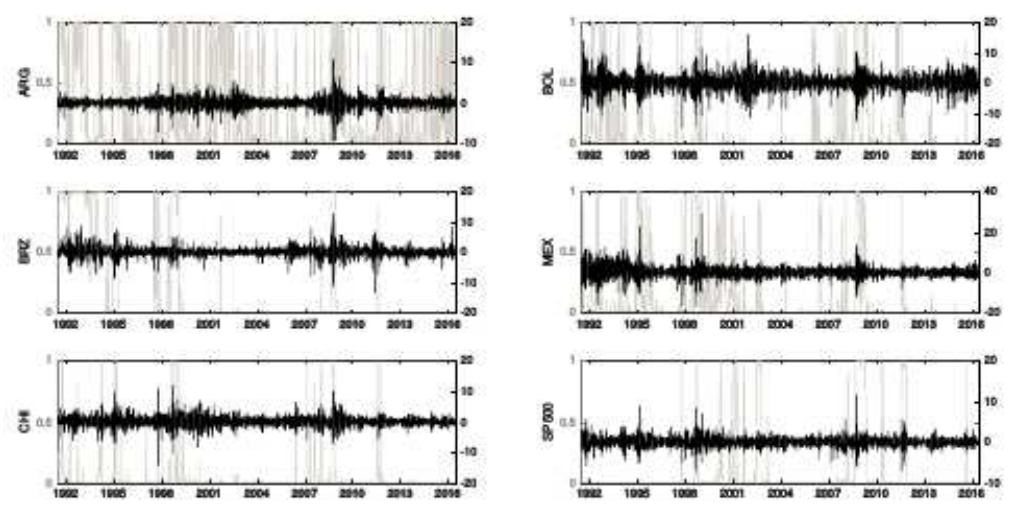

Figure 2: Smoothed probabilities of high volatility
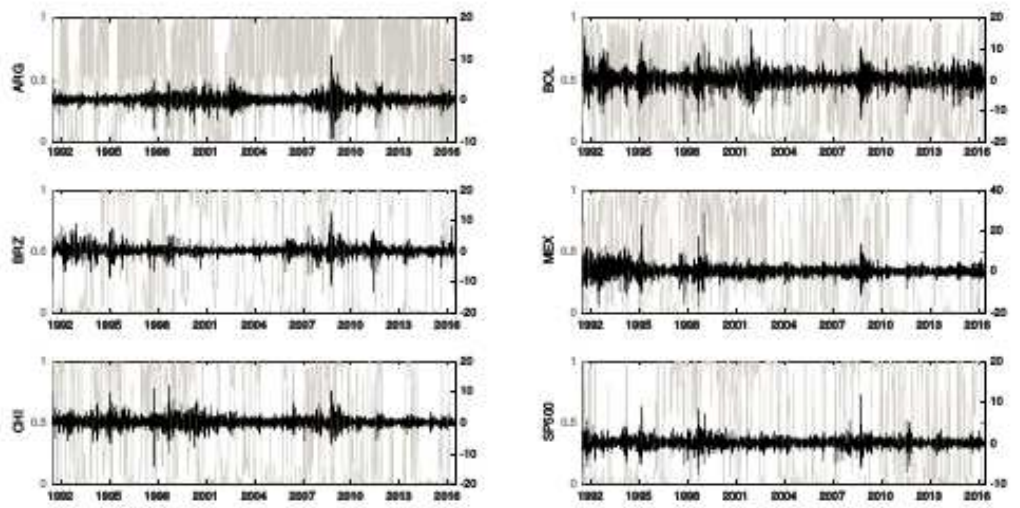

Figure 3: Smoothed probabilities of medium volatility

In Fig. 5 we plot the concordance index versus the Pearson sample correlation. Even though the sample correlations are lower than the concordance measures, the order of relevance is almost the same. However, the Pearson sample correlation is not appropriate since the random variable is measured in a discrete finite space.

For all the MCMC output and each pairwise of indexes, we compute the 

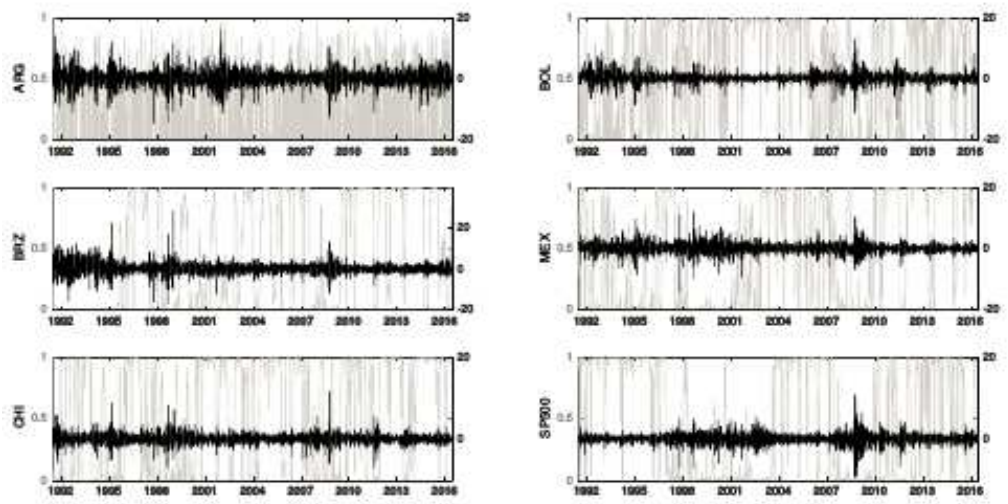

Figure 4: Smoothed probabilities of low volatility

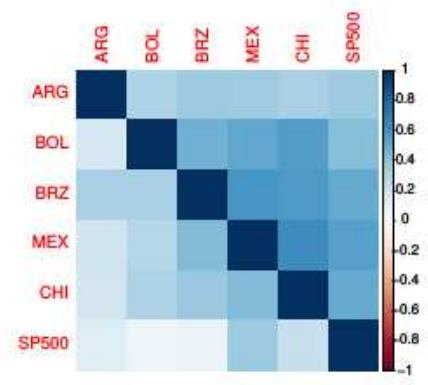

Figure 5: Correlogram of concordance index (bottom) and Pearson correlation (top)

perfect synchronization measure suggested by Hading and Pagan. In Table. 4 we present the mean versus the standard error for this measure. The standard errors show the good accuracy of our estimation procedure. The higher synchronization are for MEX-CH, BRZ-MEX and BRZ-CH.

In Fig. 6 we plot the perfect synchronization in each state versus the sample correlations. As we note, there are important differences between the Harding and Pagan's measure and the Pearson correlations for each state. Most importantly, the high perfect synchronization are in the periods of high volatility.

In Table 5, we compute the probabilities of the state $j$ for the index $r$, conditional to the state $i$ for the index $s$, for all the indexes $r \neq s$ and all the states $i, j=1,2,3$. The bold diagonal to each pairwise conditional probabilities, is the perfect synchronization in these pair of indexes. That is, it coincides with 
Table 4: Mean of the Harding and Pagan Concordance index versus its standard error

\begin{tabular}{c|cccccc} 
SE/Mean & ARG & BOL & BRZ & MEX & CHI & SP500 \\
\hline ARG & 1.0000 & 0.3077 & 0.3642 & 0.3597 & 0.3091 & 0.3529 \\
BOL & 0.0163 & 1.0000 & 0.4887 & 0.5173 & 0.5521 & 0.4308 \\
BRZ & 0.0193 & 0.0204 & 1.0000 & 0.5875 & 0.5555 & 0.5036 \\
MEX & 0.0265 & 0.0215 & 0.0233 & 1.0000 & 0.6102 & 0.5369 \\
CHI & 0.0162 & 0.0161 & 0.0194 & 0.0334 & 1.0000 & 0.5095 \\
SP500 & 0.0114 & 0.0116 & 0.0166 & 0.0193 & 0.0128 & 1.0000 \\
\hline
\end{tabular}

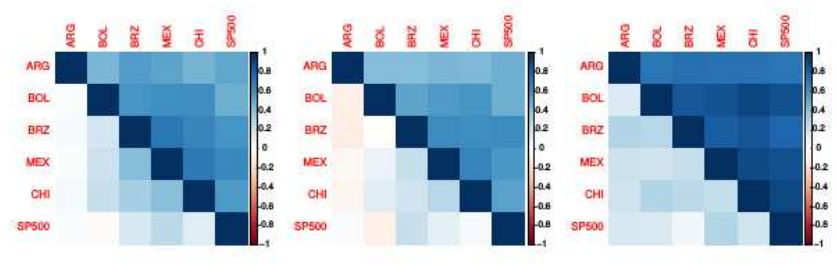

Figure 6: Correlograms of concordance index of low (left), medium (center) and high (right) volatility

Harding and Pagan index of concordance. However, we computed them for all concordance in every state. The higher synchronization in low volatility indexes is given by MEX and CHI (0.37); BOL and CHI (0.36) and, MEX and CHI with the SP500 (for 0.30 and 0.31 probabilities).

The markets in concordance for high volatility are: BRZ and ARG (0.09); ARG and MEX (0.06); ARG and BOL, ARG and SP500, BOL and BRZ and MEX and BRZ (all with 0.04 probabilities). The higher probabilities are given for the most of pairwise conditional probabilities of medium volatility to every pair of indexes. For instance, in 1996 the six indexes stay in high, medium and low volatility in $1 \%, 39 \%$ and $60 \%$ of the daily periods of the time. In 2008 , the $30 \%, 52 \%$ and $18 \%$ in high, medium and low and, in the $20158 \%, 27 \%$ and $64 \%$ respectively.

By using the posterior moments of Table 3, we compute for each index the posterior smoothed probabilities and simulate the hidden indicators of the states. We use the forward-backward filtering algorithm (see, [5], [30], [24], and [15]). In general, once we have compute the smoothed probabilities for all $t$ (forward filtering). By using random uniform numbers and the posterior transition matrices, we simulate the hidden indicator of the states from $T$ to 
$t=1$ (backward filtering) for each index.

In Figs. 7, 8 to 9, we present the number of days that every index stays in high, medium and low volatilities. These figures show that the days in medium volatilities are more prominent in almost every index (exception is ARG). However, the days in high volatility are the lowest.
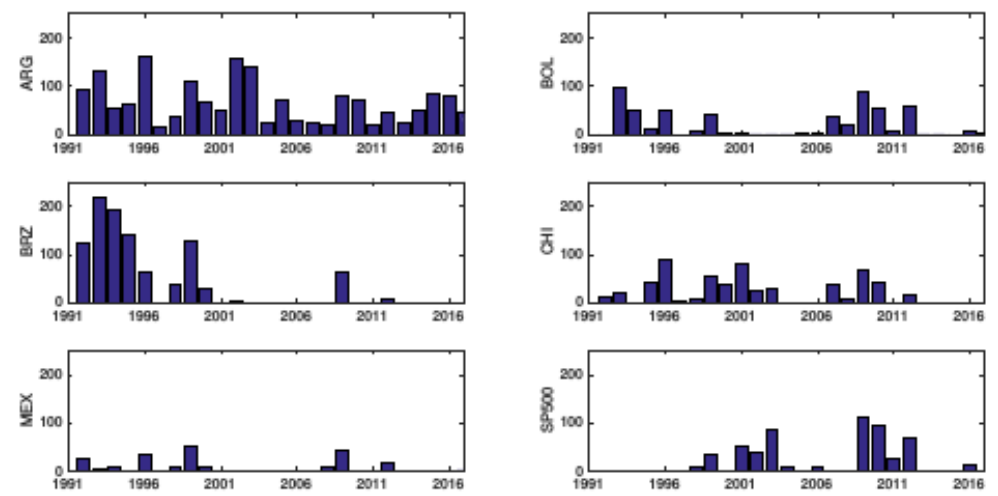

Figure 7: Days of high volatility according to the posterior probability of each model
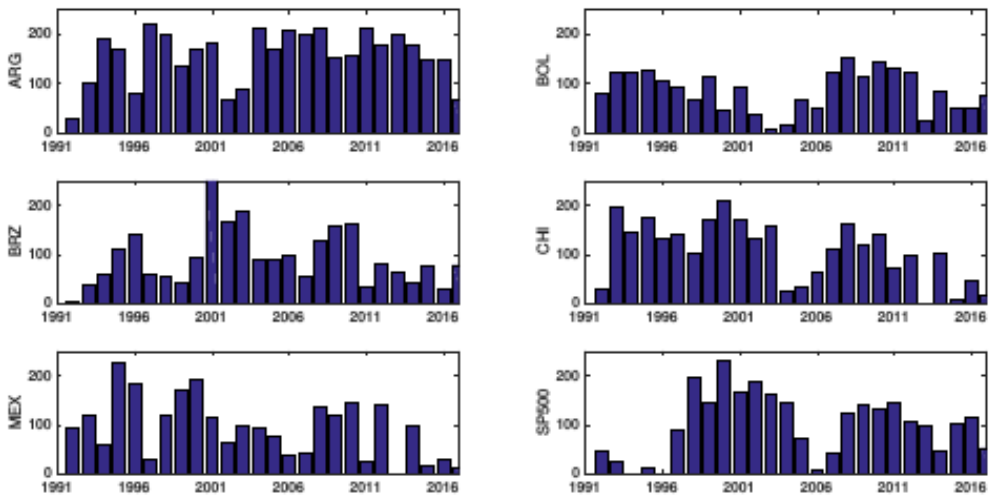

Figure 8: Days of medium volatility according to the posterior probability of each model

However, the days that each index stay in every state is not a relative a measure. Therefore, in Fig. 10 we weight the days that all the Latin American 

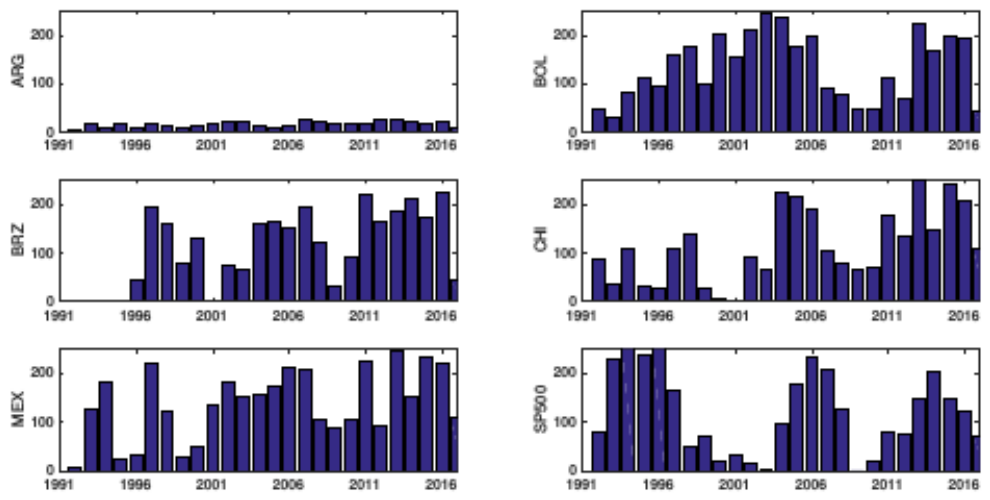

Figure 9: Days of low volatility according to the posterior probability of each model

indexes are synchronized in every year, by the percentage of time that each volatility is given (left plots). In this case, the high of the bar gives the strong of the synchronization. In 1995 (Mexican crisis), in 1999 (Russian crisis) and in the prime crisis in 2008 the high volatility is strongly synchronized than periods of low volatility. In the right plots in Fig. 10, the high volatilities are only synchronized in periods of time in the 1999 and the 2008 years. In summary, in these weighted plots, the low, medium and high common periods of time of different volatilities can be comparable, since they are relatives frequencies more than only concordance days. The relevance of perfect synchronization by the concordance index can be ordered as: medium, high and low volatility.

The perfect synchronization measure might change over periods of time and each state. In Fig. 11 we plot the dynamics of the perfect synchronization suggested by Harding and Pagan. That is, for each pairwise of indexes and every year, we compute the concordance index. From this plot, ARG is the worst synchronized index, we denote it with bold black line in the rest of plots. From the rest of plots, BOL and MEX seem to increase the synchronization (except with the ARG index with black line). The CHI, BRZ and the SP500 do not follow a clear increasing in the synchronization paths.

On the other hand, in Fig. 12 we compute the perfect synchronization for all the Latin American indexes and all the indexes including the SP500. The black line denotes when all the indexes are perfectly synchronized. We note that, in 1997 and 2008, there are the high periods of synchronization which coincide with the Russian and the sub-prime crisis. The blue line, is the perfect 

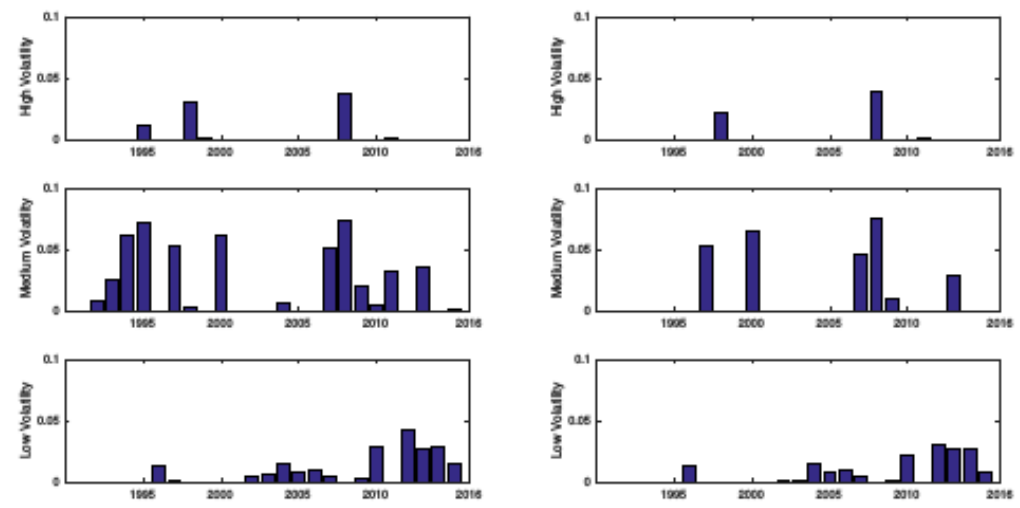

Figure 10: Weighted days per year of perfect synchronization: Latin America (left) and all indexes (right)
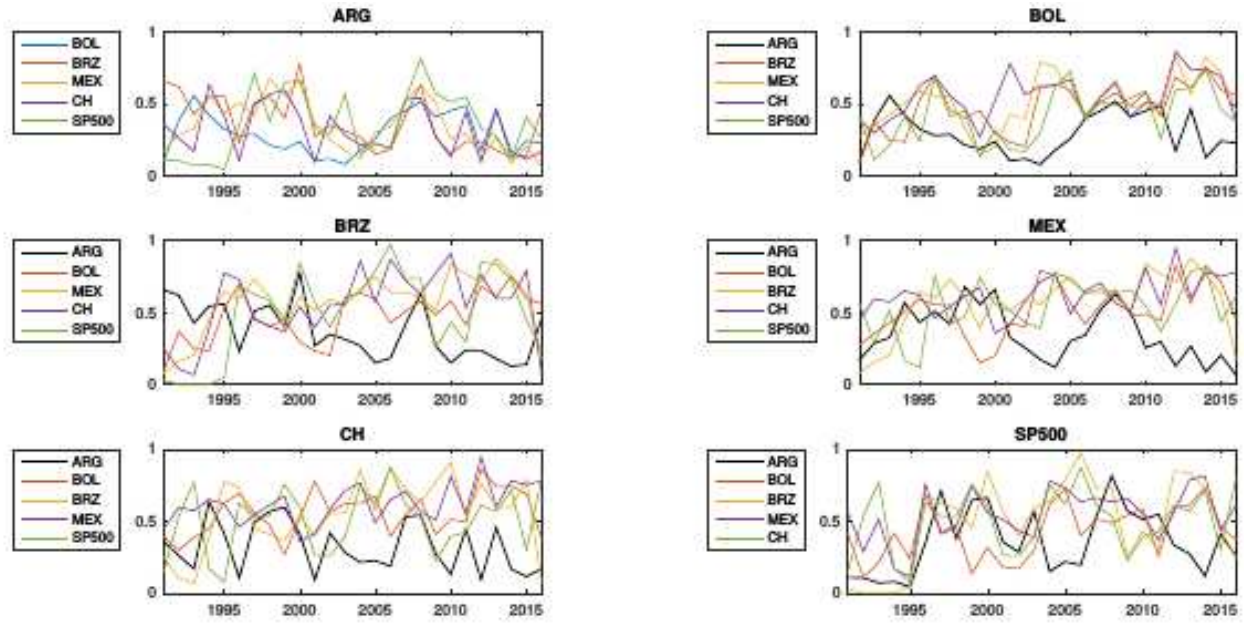

Figure 11: Concordance Index

synchronization for all the LA indexes. In the same way, the Mexican crisis in 1995 synchronizes the the indexes except the SP500 as expected.

To analyze a possible cluster in these indexes, we plot the linkage over the Euclidean distances between the all the growth rates and the posterior hidden indicator of the states. In Fig 13 we plot both in a dendrogram. The hidden indicators of the states, cluster CHI and MEX close to SP500 followed by BOL. The clustering measure of the hidden indicators of the states than clustering 


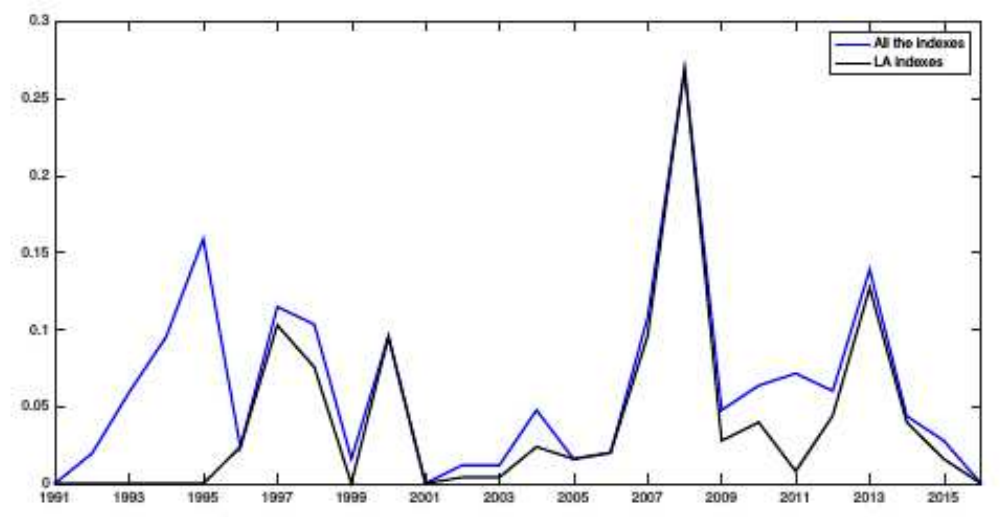

Figure 12: High volatility

by the growth rates are not very different.
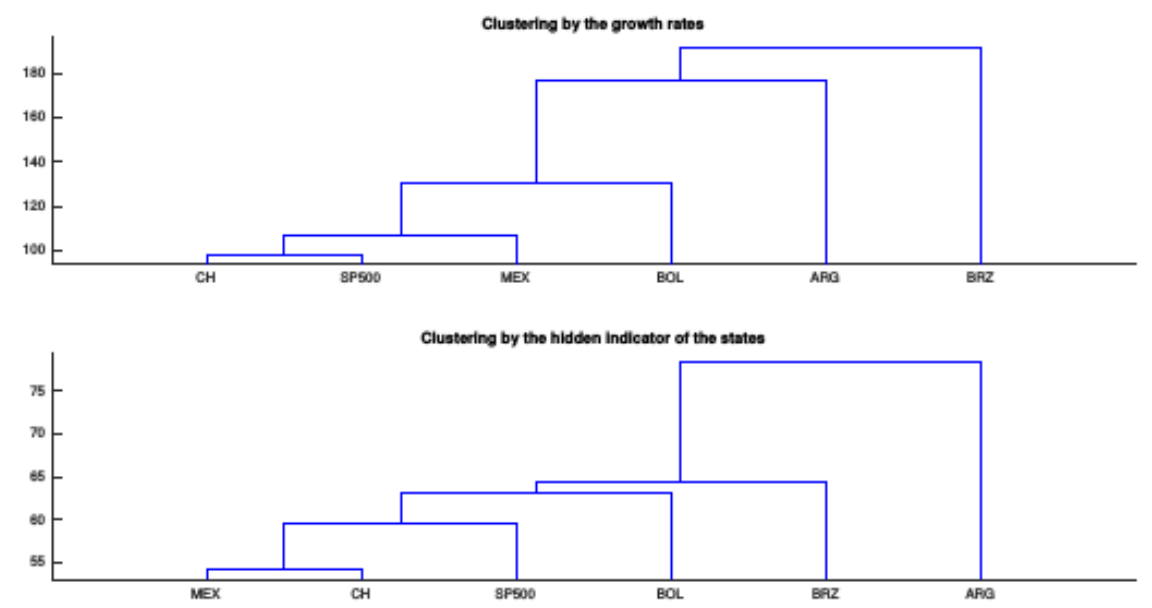

Figure 13: Clustering by a dendrogram plot

\section{Conclusions}

In this paper, we have studied the synchronization in the Latin American's stock exchange indexes and the S\&P 500 index. We used data for daily returns 
from 1992 to 2016. We modeled the data of each index using an autoregressive process subject to Markov switching parameters. To solve the model, we used the Marginal Likelihood simulation approach suggested by [15]. That is, the parameters are subject to regime switching and the number of lags and number of states are unknown for every index. We found that every index is characterized by a three state Markov switching model. We also studied the synchronization for these changes in volatilities over time. Once we chose the best Markov switching model to represent the data, we simulated the posterior indicator of the states as the associated smoothed probabilities to each state dependent volatility. Under the appropriate model selection, we studied possible synchronizations for all indexes.

By using measures of synchronization as the [18] concordance index, conditional probabilities, features and duration of the states, we found the following: MEX and CHI are the strongest synchronized markets; the synchronization of BOL and MEX has increased over time; ARG is the most nonsynchronized index; periods of high volatility coincide with the Mexican (1995), Sub-prime (2008-2009) and Russian (1999) crisis for all Latin American indexes; the stronger synchronization is given in periods of medium and high volatility; the less volatile index, is the SP500 followed by the MEX and CHI; we provide a concordance evidence of a possible cluster for CHI, MEX and BRZ, the two first closest to SP500.

In this paper, we do not study a contagion effects since our approach relies on univariate Markov switching process. We leave the multivariate switching framework for future research.

\section{References}

[1] M.L. Aiolfi, A.V. Catão, and A. Timmermann Common factors in Latin America's business cycles, Journal of Development Economics 952 (2011), 212-228.

[2] G. Bekaert, C.R. Harvey, Emerging markets finance, Journal of Empirical Finance 10 1-2 (2003), 3-55.

[3] G. Canarella, S. Pollard, A switching ARCH (SWARCH) model of stock market volatility: some evidence from Latin America, International Review of Economics 544 (2007), 445462.

[4] C.K. Carter, R. Kohn, On Gibbs sampling for state space models, Biometrika 813 (1994), 541-553.

[5] S. Chib, Marginal Likelihood from the Gibbs Output, Journal of the American Statistical Association 90432 (1995), 1313-1321.

[6] R. Cont, Empirical properties of asset returns: stylized facts and statistical issues, Quantitative Finance 1 (2001), 223-236. 
[7] P.F. Diamandis, Financial liberalization and changes in the dynamic behaviour of emerging market volatility: Evidence from four Latin American equity markets, Research in International Business and Finance 223 (2008), 362-377.

[8] D.A. Dickey, W.A. Fuller, Likelihood ratio statistics for autoregressive time series with a unit root, Econometrica 494 (1981), 1057-1072.

[9] G. Dufrénot, V. Mignon, A.Péguin-Feissolle, The effects of the subprime crisis on the Latin American financial markets: An empirical assessment, Economic Modelling 285 (2011), 2342-2357.

[10] S. Edwards, J.G. Biscarri, F. Pérez de Gracia, Stock market cycles, financial liberalization and volatility, Journal of International Money and Finance 227 (2003), 925-955.

[11] S. Edwards, R. Susmel, Volatility dependence and contagion in emerging equity markets, Journal of Development Economics 662 (2001), 505-532.

[12] S. Frühwirt-Schnatter, Finite Mixture and Markov Switching Models by S. FRHWIRTHSCHNATTER (First ed.), New York, (2006).

[13] S. Frühwirth-Schnatter, Markov Chain Monte Carlo Estimation of Classical and Dynamic Switching and Mixture Models, Journal of the American Statistical Association 96453 (2001), 194-209.

[14] S. Frühwirth-Schnatter, Estimating marginal likelihoods for mixture and markov switching models using bridge sampling techniques, Econometrics Journal 71 (2004), 143-167.

[15] S. Frühwirth-Schnatter, Finite Mixture and Markov Switching Models (Springer Series in Statistics), (1 ed.) New York: Springer, (2006).

[16] L. Gagnon, G.A. Karolyi, Price and volatility transmission across borders, Financial Markets, Institutions and Instruments 153 (2006), 107-158.

[17] J.D. Hamilton, A new approach to the economic analysis of nonstationary time series and the business cycle, Econometrica 572 (1989), 357-84.

[18] D. Harding, A. Pagan, Macroeconomics and the Real World-Volume 1: Econometric Techniques and Macroeconomics, Chapter Knowing th, Oxford. (2000), 23-42.

[19] D. Harding, A. Pagan, Dissecting the cycle: A methodological investigation, Journal of Monetary Economics 492 (2002), 365-381.

[20] D. Harding, A. Pagan, A comparison of two business cycle dating methods, Journal of Economic Dynamics and Control 279 (2003), 1681-1690.

[21] C.R. Harvey, Predictable Risk and Returns in Emerging Markets, Review of Financial Studies 83 (1995), 773-816.

[22] K.S. Im, J. Lee, M.A. Tieslau, Festschrift in Honor of Peter Schmidt, Chapter More powerful unit root tests with non-normal errors, New York, Springer (2014), 315-342.

[23] S. Kaufmann, Measuring business cycles with a dynamic markov switching factor model: an assessment using bayesian simulation methods, Econometrics Journal 31 (2000), $39-65$.

[24] C.J. Kim, Dynamic linear models with markov-switching, Journal of Econometrics 60 1-2 (1994), 1-22.

[25] C.J. Kim, C.R. Nelson, Business cycle turning points, a new coincident index, and tests of duration dependence based on a dynamic factor model with regime switching, The Review of Economics and Statistics 802 (1998), 188-201. 
[26] D. Kunovac, Asymmetric correlations on the Croatian equity market, Financial theory and practice 351 (2011), 1-24.

[27] X.l. Meng, W.H. Wong, Simulating ratios of normalizing constants via a simple identity: A theoretical exploration, Statistica Sinica 64 (1996), 831-860.

[28] S.B. Ramos, J.K. Vermunt, J.G. Dias, When markets fall down: Are emerging markets all the same, International Journal of Finance and Economics 164 (2011), 324-338.

[29] O. Rojas, C. Trejo-Pech, Nonlinear Time Series and Finance, Chapter Financial Time Series: Stylized Facts for the Mexican Stock Exchange Index Compared to Developed Markets, Mexico: Universidad de Guadalajara (2014), 228-245.

[30] N. Shephard, Partial non-gaussian state space, Biometrika 811 (1994), 115-131.

[31] R. Susmel, Extreme observations and diversification in Latin American emerging equity markets, Journal of International Money and Finance 207 (2001), 971-986.

[32] M. Zouhair, C. Lanouar, A.N. Ajmi, Contagion versus Interdependence: The Case of the BRIC Countries During the Subprime Crises, Elsevier Inc, (2013). 
Table 5: Conditional probabilities for the hidden indicators of the states $p\left(s_{t}^{r}=j \mid s_{t}^{s}=i\right)$ for $i, j=1,2,3$

\begin{tabular}{|c|c|c|c|c|c|c|}
\hline & $S_{t}^{A R G}=1$ & $S_{t}^{A R G}=2$ & $S_{t}^{A R G}=3$ & & $S_{t}^{M E X}=1$ & $S_{t}^{M E X}=2$ \\
\hline$S_{t}^{B O L}=1$ & 0.04 & 0.04 & 0.00 & $S_{t}^{A R G}=1$ & 0.06 & 0.13 \\
\hline$S_{t}^{B O L}=2$ & 0.12 & 0.22 & 0.02 & $S_{t}^{A R G}=2$ & 0.04 & 0.26 \\
\hline$S_{t}^{B O L}=3$ & 0.11 & 0.40 & 0.04 & $S_{t}^{A R G}=3$ & 0.00 & 0.03 \\
\hline$S_{t}^{B R Z}=1$ & 0.09 & 0.06 & 0.01 & $S_{t}^{B O L}=1$ & 0.03 & 0.04 \\
\hline$S_{t}^{B R Z}=2$ & 0.14 & 0.24 & 0.02 & $S_{t}^{B O L}=2$ & 0.05 & 0.17 \\
\hline$S_{t}^{B R Z}=3$ & 0.05 & 0.36 & 0.03 & $S_{t}^{B O L}=3$ & 0.03 & 0.20 \\
\hline$S_{t}^{M E X}=1$ & 0.06 & 0.04 & 0.00 & $S_{t}^{B R Z}=1$ & 0.04 & 0.07 \\
\hline$S_{t}^{M E X}=2$ & 0.13 & 0.26 & 0.03 & $S_{t}^{B R Z}=2$ & 0.06 & 0.22 \\
\hline$S_{t}^{M E X}=3$ & 0.10 & 0.35 & 0.04 & $S_{t}^{B R Z}=3$ & 0.00 & 0.12 \\
\hline$S_{t}^{C H}=1$ & 0.03 & 0.01 & 0.00 & $S_{t}^{C H}=1$ & 0.02 & 0.02 \\
\hline$S_{t}^{C H}=2$ & 0.13 & 0.25 & 0.02 & $S_{t}^{C H}=2$ & 0.06 & 0.24 \\
\hline$S_{t}^{C H}=3$ & 0.12 & 0.39 & 0.04 & $S_{t}^{C H}=3$ & 0.02 & 0.16 \\
\hline$S_{t}^{S P 500}=1$ & 0.04 & 0.03 & 0.00 & $S_{t}^{S P 500}=1$ & 0.03 & 0.05 \\
\hline$S_{t}^{S P 500}=2$ & 0.12 & 0.28 & 0.03 & $S_{t}^{S P 500}=2$ & 0.05 & 0.21 \\
\hline$S_{t}^{S P 500}=3$ & 0.12 & 0.34 & 0.04 & $S_{t}^{S P 500}=3$ & 0.03 & 0.16 \\
\hline & $S_{t}^{B O L}=1$ & $S_{t}^{B O L}=2$ & $S_{t}^{B O L}=3$ & & $S_{t}^{C H}=1$ & $S_{t}^{C H}=2$ \\
\hline$S_{t}^{A R G}=1$ & 0.04 & 0.12 & 0.11 & $S_{t}^{A R G}=1$ & 0.03 & 0.13 \\
\hline$S_{t}^{A R G}=2$ & 0.04 & 0.22 & 0.40 & $S_{t}^{A R G}=2$ & 0.01 & 0.25 \\
\hline$S_{t}^{A R G}=3$ & 0.00 & 0.02 & 0.04 & $S_{t}^{A R G}=3$ & 0.00 & 0.02 \\
\hline$S_{t}^{B R Z}=1$ & 0.04 & 0.09 & 0.03 & $S_{t}^{B O L}=1$ & 0.02 & 0.04 \\
\hline$S_{t}^{B R Z}=2$ & 0.02 & 0.14 & 0.23 & $S_{t}^{B O L}=2$ & 0.02 & 0.18 \\
\hline$S_{t}^{B R Z}=3$ & 0.02 & 0.14 & 0.29 & $S_{t}^{B O L}=3$ & 0.00 & 0.19 \\
\hline$S_{t}^{M E X}=1$ & 0.03 & 0.05 & 0.03 & $S_{t}^{B R Z}=1$ & 0.03 & 0.08 \\
\hline$S_{t}^{M E X}=2$ & 0.04 & 0.17 & 0.20 & $S_{t}^{B R Z}=2$ & 0.01 & 0.21 \\
\hline$S_{t}^{M E X}=3$ & 0.02 & 0.14 & 0.32 & $S_{t}^{B R Z}=3$ & 0.00 & 0.12 \\
\hline$S_{t}^{C H}=1$ & 0.02 & 0.02 & 0.00 & $S_{t}^{M E X}=1$ & 0.02 & 0.06 \\
\hline$S_{t}^{C H}=2$ & 0.04 & 0.18 & 0.19 & $S_{t}^{M E X}=2$ & 0.02 & 0.24 \\
\hline$S_{t}^{C H}=3$ & 0.02 & 0.17 & 0.36 & $S_{t}^{M E X}=3$ & 0.00 & 0.11 \\
\hline$S_{t}^{S P 500}=1$ & 0.02 & 0.03 & 0.03 & $S_{t}^{S P 500}=1$ & 0.01 & 0.05 \\
\hline$S_{t}^{S P 500}=2$ & 0.03 & 0.14 & 0.26 & $S_{t}^{S P 500}=2$ & 0.01 & 0.18 \\
\hline$S_{t}^{S P 500}=3$ & 0.04 & 0.19 & 0.27 & $S_{t}^{S P 500}=3$ & 0.01 & 0.17 \\
\hline & $S_{t}^{B R Z}=1$ & $S_{t}^{B R Z}=2$ & $S_{t}^{B R Z}=3$ & & $S_{t}^{S P 500}=1$ & $S_{t}^{S P 500}=2$ \\
\hline$S_{t}^{A R G}=1$ & 0.09 & 0.14 & 0.05 & $S_{t}^{A R G}=1$ & 0.04 & 0.12 \\
\hline$S_{t}^{A R G}=2$ & 0.06 & 0.24 & 0.36 & $S_{t}^{A R G}=2$ & 0.03 & 0.28 \\
\hline$S_{t}^{A R G}=3$ & 0.01 & 0.02 & 0.03 & $S_{t}^{A R G}=3$ & 0.00 & 0.03 \\
\hline$S_{t}^{B O L}=1$ & 0.04 & 0.02 & 0.02 & $S_{t}^{B O L}=1$ & 0.02 & 0.03 \\
\hline$S_{t}^{B O L}=2$ & 0.09 & 0.14 & 0.14 & $S_{t}^{B O L}=2$ & 0.03 & 0.14 \\
\hline$S_{t}^{B O L}=3$ & 0.03 & 0.23 & 0.29 & $S_{t}^{B O L}=3$ & 0.03 & 0.26 \\
\hline$S_{t}^{M E X}=1$ & 0.04 & 0.06 & 0.00 & $S_{t}^{B R Z}=1$ & 0.02 & 0.02 \\
\hline$S_{t}^{M E X}=2$ & 0.07 & 0.22 & 0.12 & $S_{t}^{B R Z}=2$ & 0.06 & 0.22 \\
\hline$S_{t}^{M E X}=3$ & 0.04 & 0.12 & 0.32 & $S_{t}^{B R Z}=3$ & 0.01 & 0.18 \\
\hline$S_{t}^{C H}=1$ & 0.03 & 0.01 & 0.00 & $S_{t}^{M E X}=1$ & 0.03 & 0.05 \\
\hline$S_{t}^{C H}=2$ & 0.08 & 0.21 & 0.12 & $S_{t}^{M E X}=2$ & 0.05 & 0.21 \\
\hline$S_{t}^{C H}=3$ & 0.05 & 0.17 & 0.33 & $S_{t}^{M E X}=3$ & 0.00 & 0.17 \\
\hline$S_{t}^{S P 500}=1$ & 0.02 & 0.06 & 0.01 & $S_{t}^{C H}=1$ & 0.01 & 0.01 \\
\hline$S_{t}^{S P 500}=2$ & 0.02 & 0.22 & 0.18 & $S_{t}^{C H}=2$ & 0.05 & 0.18 \\
\hline$S_{t}^{S P 500}=3$ & 0.11 & 0.12 & 0.26 & $S_{t}^{C H}=3$ & 0.02 & 0.23 \\
\hline
\end{tabular}

